\title{
Microarray comparison of the gene expression profiles in the adult vs. embryonic day 14 rat liver
}

\author{
JIE ZHENG ${ }^{1 *}$, SHUNA YU ${ }^{2 *}$, ZHENGCHEN JIANG $^{3}$, CAIXING SHI $^{2}$, \\ $\mathrm{JIN} \mathrm{LI}^{2}$, XIAODONG DU ${ }^{2}$, HAILIANG WANG ${ }^{3}$ and JIYING JIANG ${ }^{2}$ \\ Departments of ${ }^{1}$ Pathology, ${ }^{2}$ Anatomy, and ${ }^{3}$ Clinical Medicine, Weifang Medical University, \\ Weifang, Shandong 261053, P.R. China
}

Received April 21, 2014; Accepted June 13, 2014

DOI: 10.3892/br.2014.303

\begin{abstract}
The aim of the present study was to identify the differentially-expressed genes of embryonic day 14 (ED 14) rat liver in comparison to adult rat liver, which may provide specific information for the investigation of the hepatogenesis mechanism. The gene expression profiles of ED 14 and adult rat livers were investigated using microarray analysis (the Illumina RatRef-12 Expression BeadChip). Quantitative polymerase chain reaction (qPCR) analyses were conducted to confirm the gene expression. There were 787 genes upregulated in the embryonic liver. Based on the gene ontology classification system, which was analyzed by the database for annotation, visualization and integrated discovery software, a number of the upregulated genes were categorized into the distinct and differentially-expressed functional groups, including metabolism pathway, cell cycle, transcription, signal transduction, purine metabolism, cell structure, transportation and apoptosis. qPCR analyses confirmed the gene expression. Eleven upregulated genes were found in the ED 14 rat liver, which may provide specific information for the understanding of the molecular mechanisms that control hepatogenesis. These overexpressed genes are potential markers for identifying hepatic progenitor cells.
\end{abstract}

\section{Introduction}

The liver is the largest internal organ that is involved in a vast number of essential biochemical functions, including metabolic, exocrine and endocrine systems. Hepatogenesis is the process of liver formation, and the liver originates

Correspondence to: Professor Jiying Jiang, Department of Anatomy, Weifang Medical University, 7166 Baotong Street, Weifang, Shandong 261053, P.R. China

E-mail: jiangjiying2014@126.com

*Contributed equally

Key words: rat liver, hepatogenesis, microarray, quantitative polymerase chain reaction from the gut endoderm. In rats, hepatic development begins at embryonic day (ED) 9.5, as primitive epithelial cells of the foregut come into contact with the cardiac mesoderm and form the liver diverticulum. Subsequently, these cells invade the septum transversum, proliferate extensively and differentiate further (1). At ED 10.5, these cells acquire the morphological appearance of immature liver epithelial cells and are termed hepatoblasts, which are bipotential and capable of generating hepatocyte and cholangiocyte cell lineages $(1,2)$. Germain et al (3) reported that hepatoblasts began to differentiate to hepatocyte and cholangiocyte cells at ED 15 in rats; however, Petkov et al (4) found that differentiation started at ED 16-17. Previous studies have found that certain molecular mechanisms regulate hepatogenesis, such as cardiac tissue induction and the role of the septum transversum mesenchyme, as well as the presence of endothelial cells (5-7). Several transcription factors are also involved in controlling distinct aspects of hepatogenesis, including Prox 1, which is necessary for hepatoblast migration (8); homeobox factor Hex, which is essential for morphogenesis and growth of the liver bud (9); HNF4, which is required for hepatocyte differentiation and epithelial transformation of the liver (10); and GATA6, which is required for liver bud growth (11).

Hepatogenesis is an extremely intricate process, as a variety of genes are involved and a complex network is formed. However, certain mechanisms are not clear; numerous associated genes have not been found. Future studies are required to investigate how to get the endoderm reactive potency in liver development, and to examine whether the upstream and downstream activation mechanisms of the genes are involved. The interactions between the epithelial and mesenchymal cells remain unclear.

In the present study, a rat genome-wide gene expression bead chip was used to investigate the global patterns of gene expression of the rat liver at ED 14 compared to adult rat liver, which may provide specific information for the investigation of hepatogenesis mechanism. The Illumina BeadChip is a forefront chip technology, and its genome expression chip can be used for human, mouse and rat genome-wide expression studies $(12,13)$. The rat genome microarray used could detect the expression of 21,910 genes. However, in our previous study (4), the microarray only focused on a portion of the genes expressed during rat hepatogenesis. Therefore, 
the current study involves the genome-wide analysis of differentially-expressed genes.

\section{Materials and methods}

Animals and treatment. Two pregnant Sprague-Dawley rats at ED 14 (experimental group) and two adult female Sprague-Dawley rats (control group) were purchased from the Experimental Animal Center in Shandong University of Traditional Chinese Medicine (Shandong, China). The livers of the rats were removed. All the experimental animals used in the study were utilized under the protocol approved by the Institutional Animal Care and Use Committee of Weifang Medical University (Shandong, China).

Isolation of ED 14 and adult liver cells. The cell suspensions from the ED 14 and adult rat livers were prepared as previously reported (14). The cells were plated in gelatin-coated dishes at a density of $15 \times 10^{6}$ cells per $10-\mathrm{cm}$ plate. Following the removal of the hematopoietic cells (no attachment) by washing with phosphate-buffered saline, the epithelial cells were allowed to attach for $16 \mathrm{~h}$.

RNA extraction and quality assessment. Total RNA was isolated from frozen liver cells by using TRIzol reagent (Invitrogen, Carlsbad, CA, USA) in accordance with the manufacturer's instructions. The samples were digested with DNase (Invitrogen) and eluted with $30 \mu \mathrm{l}$ RNase-free water. The concentration and quality of the samples were measured by using the DU-640 nucleic acid/protein analyzer (Beckman Coulter, Brea, CA, USA).

Microarray analysis procedures. The microarray experiments (including sample labeling, hybridization and initial data analysis) were performed at Beijing Emei Tongde Technology Development Co., Ltd. (Beijing, China). For each sample, biotinylated cRNA was prepared using an Ambion Illumina TotalPrep RNA amplification kit (Applied Biosystems, Foster City, CA, USA). Total RNA (5 $\mu \mathrm{g})$ was converted to double-stranded cDNA using T7-oligo (dT) primers. Subsequently, an in vitro transcription reaction was performed to amplify biotinylated cRNA, as described in the manufacturer's instructions (Illumina, Inc., San Diego, CA, USA). The biotinylated cRNA was hybridized to a RatRef-12 Expression BeadChip platform that contained 21,910 probes (Illumina, Inc.). Hybridization, washing and scanning were performed in accordance with the manufacturer's instructions.

The chips were scanned by the BeadArray reader (Illumina, Inc.). The microarray images were registered and extracted automatically during the scan using the manufacturer's default settings.

Microarray data analysis. The microarray data were analyzed with Illumina BeadStudio software (Illumina, Inc.). The average normalization method was used. The sample intensities were scaled by a factor equal to the ratio of the average intensity of the virtual sample to the average intensity of the provided sample. The background was subtracted prior to scaling. The average normalization minimizes the amount of variation resulting from constant multiplicative factors (including scanner power). The sample intensities were scaled by a factor so that the average signal of all the samples was equal to the global average of all the sample signals. Background subtraction was performed prior to scaling, and therefore, one-half of the unexpressed targets were predicted to have negative signals.

The gene expression differences between the two groups were calculated by DiffScore. The screening criteria for the differences in gene expression were that the detection P-value of the experimental or control group was $<0.01$ and the experimental group DiffScore value was $>20$.

Gene ontology (GO) analysis was performed with the GO classification system and using the database for annotation, visualization and integrated discovery software (http://david. abcc.ncifcrf.gov/) (15). The over-representation of the genes with altered expression within specific GO categories was determined using the one-tailed Fisher's exact probability test, which was modified by adding a jack-knifing procedure. This penalizes the significance of categories having extremely few (one or two) genes and favors more robust categories having a larger number of genes (16). A number of these upregulated genes could be categorized into distinct, differentially-expressed functional groups (including the genes that are associated with the metabolism pathway, the cell cycle, transcription, signal transduction, urine metabolism, cell structure, transportation and apoptosis).

Quantitative polymerase chain reaction ( $q P C R$ ) experiment. To validate the microarray results, the 11 most differentially and significantly expressed genes were selected for qPCR analysis using the same RNA samples that were used for the microarray. The ABI PRISM 7700 sequence detection system (Applied Biosystems) was used to perform one-step qPCR. The results were quantified as $\mathrm{Ct}$ values; this value signifies relative gene expression (the ratio of target/control). qPCR results were analyzed by the comparative $\mathrm{Ct}$ method (17). Table I presents the primers used in the study. All the results are expressed as mean \pm standard deviation. Statistical analysis was performed with analysis of variance followed by Student's t-test using SPSS 11.0 (SPSS, Inc., Chicago, IL, USA).. P<0.05 was considered to indicate a statistically significant difference.

\section{Results}

Screening of the gene expression profile of ED 14 rat liver by microarray analysis. To screen the genes associated with ED 14 rat liver, a genome-wide microarray analysis was performed using the Illumina RatRef-12 Expression BeadChip (Illumina, Inc.), which contains 21,910 distinct rat oligonucleotide probes. This microarray chip encompasses the largest number of rat genes that were available at the time of the analysis. Total RNA was extracted from ED 14 and adult rat liver cells. The extracted RNA was of high quality and was homogeneous. Data analyses by average normalization of the hybridization signals showed that the expressions of 787 genes were upregulated in the ED 14 rat liver (DiffScore values, >20). A number of these highly-expressed genes could be grouped into distinct, differentially-expressed functional groups based on the GO classification system. These upregulated genes included those that are associated with 
Table I. Oligonucleotide sequences of the primers used for quantitative polymerase chain reaction.

\begin{tabular}{lll}
\hline Gene symbol & \multicolumn{1}{c}{ Forward primer (5-3') } & Reverse primer (5-3') \\
\hline Hk2 & CTGGTGCCCGACTGTGAT & CCATTTCCACCTTCATTCTT \\
Eno2 & GGGACAAACAGCGTTACTT & CAATGTGGCGATAGAGGG \\
$P d e 9 a$ & AGCCTTCGGTGAGTCCTGG & GCTGTGCCCTTGTTCTTGC \\
KIF4 & CCACCATCTCCCTTCTGA & CTCCACCACTTTGAGTCCTT \\
Plagl & TTCCACCTAAGCCCAAAC & CTCCTCCTCAGCCACAGA \\
Orcl & CCTTGCCTTCCAGCGAACT 2 & CGCCACCTTGTAACTCCATCAG \\
Foxm1 & GGATGATGCCGTCCAGTT & CACCACGCTGATGGGAAA \\
Ccnf & GTAATGTGACGGATAGAACG & GTAAATGGCTGGGAAGAG \\
SPAG5 & ATCGCTACTTGACATTGGA & CTCAGGATTGGGTCGTTT \\
& ACACCCACCGCAGAACTA & TCTCCTGGTCTCCCTCAT \\
& TGTAAAGGCCAAATAGAAC & ACAATGGGAATGCTGACT \\
& AGACAGCCGCATCTTCTTGT & CTTGCCGTGGGTAGAGTCAT
\end{tabular}

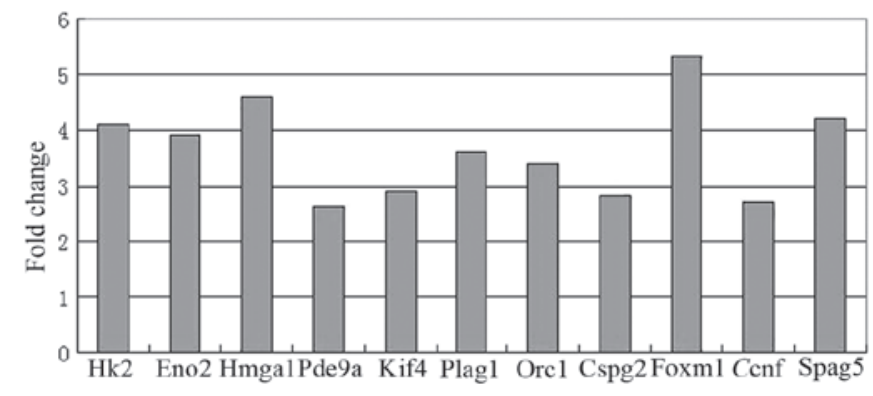

Figure 1. Fold changes for the expression of genes that are differentially expressed in rat liver at embryonic day 14 compared to adult rat liver. Fold changes were measured by quantitative polymerase chain reaction analysis of the 11 candidate genes initially selected by microarray analysis.

the metabolism pathway, the cell cycle, transcription, signal transduction, purine metabolism, cell structure, transportation and apoptosis (Table II).

$q P C R$ analysis. Fig. 1 shows the results of the qPCR experiment for 11 genes. The expression of the 11 genes were all significantly increased in ED 14 rat liver $(\mathrm{P}<0.05)$, compared to their expression in adult rat liver, confirming the validity of the microarray results.

\section{Discussion}

In the present study, the gene expression pattern of ED 14 rat liver compared to adult rat liver was investigated. The microarray analysis initially suggested that there were 787 candidate genes. Some of the identified genes are known to be involved in hepatogenesis. A previous study reported that high levels of Bmp4 expression within the septum transversum mesenchyme implied a significant role for Bmp4 signaling during hepatogenesis (5). A number of these highly-expressed genes can be grouped into distinct, differentially-expressed functional groups based on the GO classification system. These upregulated genes include genes that are associated with the metabolism pathway, cell cycle, transcription, signal transduction, purine metabolism, cell structure, transportation and apoptosis. qPCR confirmed that 11 genes were significantly and differentially expressed.
These differentially-expressed genes may provide certain information for the investigation of hepatogenesis mechanism.

In the adult rat liver, numerous genes belonging to the group of genes associated with the metabolism pathway were expressed at extremely low levels or were not expressed. However, the function of certain genes remains unknown. The Hk2, Eno2, Enol, Galnt7, Slcla3, Galnt1 and Galnt3 genes are involved in glucose metabolism. Hk2 can catalyze glucose, mannose, glucosamine and fructose into their respective 6-phosphate form through an irreversible phosphorylation reaction. Enolase (also known as phosphoglycerate hydrolase) is a critical enzyme for adenosine triphosphate synthesis in glycolysis, and is responsible for catalyzing the conversion of 2-phosphoglycerate to phosphoenolpyruvate and in catalyzing the reverse reaction during gluconeogenesis (18). Eno1 exists in the majority of tissues. However, recent studies have shown that Enol has a transcriptional regulatory function as it can combine with the c-Myc promoter region, which can inhibit c-Myc transcriptional function. The present study found increased Enol expression in the embryonic liver. This action may be associated with increased glycolysis in the embryonic liver tissue. Eno2 (also known as NSE) is the homodimer of $\gamma$ subunits and is primarily found in neurons and neuroendocrine tissues.

An example of a gene associated with the cell cycle is cyclin F, which belongs to the F-box protein family that is characterized by a $\sim 40$-amino acid motif. In contrast to the majority of cyclins, it does not bind or activate any cyclin-dependent kinases. However, cyclin F, similar to other cyclins, oscillates during the cell cycle with protein levels; it peaks in the G2 phase. Through its ability to restrain the expression of $\mathrm{CP} 110$, cyclin $\mathrm{F}$ plays a significant role in limiting centrosome duplication and in maintaining chromosome stability (19).

A spindle-binding protein, SPAG5 (also known as astrin, hMAP126), plays an important role in the regulation of mitosis. SPAG5 is located in the centromere during the medium term of mitosis. The downregulation of SPAG5 expression results in spindle deformity and may delay mitosis and therefore result in cell cycle arrest at the G2/M phase (20). Glycogen synthase kinase $3 \beta$ (GSK3 $\beta$ ) binds with SPAG5 and subsequently phosphorylates and regulates the location of SPAG5 in the spindle 
Table II. Genes with increased expression in the embryonic day 14 rat liver $^{\mathrm{a}}$.

\begin{tabular}{|c|c|c|c|c|}
\hline $\begin{array}{l}\text { Gene } \\
\text { symbol }\end{array}$ & Definition & Accession no. & $\begin{array}{l}\text { Detection } \\
\text { P-value }\end{array}$ & DiffScore \\
\hline \multicolumn{5}{|c|}{$\begin{array}{l}\text { Metabolism } \\
\text { pathway }\end{array}$} \\
\hline$H k 2$ & Hexokinase 2 & NM_012735.1 & 0 & 360.045 \\
\hline Eno2 & Eenolase $2, \gamma$ & NM_139325.1 & 0 & 360.045 \\
\hline Galnt7 & $\begin{array}{l}\text { UDP-N-acetyl- } \alpha \text {-D-galactosamine:polypeptide } \\
\mathrm{N} \text {-acetylgalactosaminyltransferase } 7\end{array}$ & NM_022926.1 & 0.00121 & 106.51 \\
\hline Gcntl & Glucosaminyl (N-acetyl) transferase 1 , core 2 & XM_579453.1 & 0.00121 & 88.097 \\
\hline$H s d 11 b 2$ & Hydroxysteroid 11- $\beta$ dehydrogenase 2 & NM_017081.1 & 0.00121 & 86.04 \\
\hline Sphkl & Sphingosine kinase 1 & NM_133386.2 & 0.00242 & 79.566 \\
\hline Galntl1 & $\begin{array}{l}\text { UDP-N-acetyl- } \alpha \text {-D-galactosamine:polypeptide } \\
\mathrm{N} \text {-acetylgalactosaminyltransferase-like } 1 \text {, transcript } \\
\text { variant } 2 \text { (predicted) }\end{array}$ & XM_001053416.1 & 0 & 74.865 \\
\hline Galnt3 & $\begin{array}{l}\text { UDP-N-acetyl- } \alpha \text {-D-galactosamine:polypeptide } \\
\mathrm{N} \text {-acetylgalactosaminyltransferase } 3\end{array}$ & NM_001015032.2 & 0.00242 & 53.1 \\
\hline Enol & Enolase $1, \alpha$ & NM_012554.1 & 0.00242 & 48.033 \\
\hline Gadl & Glutamic acid decarboxylase 1 & NM_017007.1 & 0.00121 & 34.698 \\
\hline Gfptl & Glutamine fructose-6-phosphate transaminase 1 & NM_001005879.1 & 0.00242 & 31.043 \\
\hline$D b h$ & Dopamine $\beta$ hydroxylase & NM_013158.1 & 0.00364 & 24.062 \\
\hline \multicolumn{5}{|l|}{ Cell cycle } \\
\hline$C d c 23$ & Cell division cycle 23 , yeast, homolog & XM_214588.4 & 0.00242 & 67.5 \\
\hline Ccnel & Cyclin E & XM_574426.1 & 0.00242 & 58.898 \\
\hline Ccnf & Cyclin F & XM_001054372.1 & 0 & 360.045 \\
\hline$E 2 f 1$ & E2F transcription factor 1 & XM_230765.4 & 0.00121 & 130.672 \\
\hline $\operatorname{Tg} f \beta 3$ & Transforming growth factor, $\beta 3$ & NM_013174.1 & 0.00242 & 22.983 \\
\hline Mapk8 & Mitogen-activated protein kinase 8 & XM_341399.2 & 0.00242 & 28.211 \\
\hline SPAG5 & Sperm-associated antigen 5 & XM_340848.3 & 0 & 360.045 \\
\hline Nfkbl & $\begin{array}{l}\text { Nuclear factor of } \kappa \text { light chain gene enhancer in } \\
\text { B-cells } 1, \text { p } 105\end{array}$ & XM_001075876.1 & 0.00242 & 34.263 \\
\hline \multicolumn{5}{|c|}{ Transcription } \\
\hline$E 2 f 1$ & E2F transcription factor 1 & XM_230765.4 & 0.00121 & 130.672 \\
\hline$H M G A 1$ & High-mobility group AT-hook 1 & NM_139327.1 & 0 & 360.045 \\
\hline Bmp4 & Bone morphogenetic protein 4 & NM_012827.1 & 0.00242 & 79.573 \\
\hline$N k x 2-5$ & NK2 transcription factor related, locus 5 (Drosophila) & NM_053651.1 & 0.00242 & 63.247 \\
\hline Rail4 & Retinoic acid induced 14 & NM_001011947.1 & 0.00121 & 108.339 \\
\hline Twistl & Twist gene homolog 1 (Drosophila) & NM_053530.2 & 0 & 143.869 \\
\hline Foxml & Forkhead box M1 & NM_031633.1 & 0 & 360.045 \\
\hline$Z f h x l b$ & Zinc finger homeobox $1 \mathrm{~b}$ & NM_001033701.1 & 0.00242 & 35.255 \\
\hline Sst & Somatostatin & NM_012659.1 & 0.00121 & 65.352 \\
\hline Myog & Myogenin & NM_017115.2 & 0.00242 & 36.437 \\
\hline$H d a c 7 a$ & Histone deacetylase 7A & XM_345868.3 & 0.00242 & 60.799 \\
\hline Lef1 & Lymphoid enhancer binding factor 1 & NM_130429.1 & 0 & 57.738 \\
\hline Runxl & Runt-related transcription factor 1 & NM_017325.1 & 0.00121 & 34.814 \\
\hline \multicolumn{5}{|c|}{$\begin{array}{l}\text { Signal } \\
\text { transduction }\end{array}$} \\
\hline Cptlc & Carnitine palmitoyltransferase $1 \mathrm{c}$ & XM_001078512.1 & 0.00242 & 49.495 \\
\hline Fbxl12 & F-box and leucine-rich repeat protein 12 & NM_001025700.1 & 0.00242 & 27.332 \\
\hline$P d e 9 a$ & Phosphodiesterase 9A & NM_138543.1 & 0 & 360.045 \\
\hline Dnmt2 & DNA methyltransferase 2 & NM_001031643.1 & 0.00242 & 40.064 \\
\hline Bmp4 & Bone morphogenetic protein 4 & NM_012827.1 & 0.00242 & 79.573 \\
\hline Fbxl12 & F-box and leucine-rich repeat protein 12 & NM_001025700.1 & 0.00242 & 27.332 \\
\hline$H M G A 1$ & High-mobility group AT-hook 1 & NM_139327.1 & 0 & 360.045 \\
\hline Hnrpal & Heterogeneous nuclear ribonucleoprotein A1 & NM_017248.1 & 0.00242 & 81.233 \\
\hline$H r c$ & Histidine-rich calcium-binding protein & NM_181369.2 & 0.00242 & 30.671 \\
\hline$Z f h x 1 b$ & Zinc finger homeobox $1 \mathrm{~b}$ & NM_001033701.1 & 0.00242 & 35.255 \\
\hline
\end{tabular}


Table II. Continued.

\begin{tabular}{|c|c|c|c|c|}
\hline $\begin{array}{l}\text { Gene } \\
\text { symbol }\end{array}$ & Definition & Accession no. & $\begin{array}{l}\text { Detection } \\
\text { P-value }\end{array}$ & DiffScore \\
\hline Cutll & Cut-like 1 (Drosophila) & XM_001070482.1 & 0.00242 & 84.871 \\
\hline Mapk8 & Mitogen-activated protein kinase 8 & XM_341399.2 & 0.00242 & 28.211 \\
\hline Ptk2 & PTK2 protein tyrosine kinase 2 & NM_013081.1 & 0.00242 & 47.335 \\
\hline Crk & v-Crk sarcoma virus CT10 oncogene homolog (avian) & NM_019302.1 & 0.00242 & 38.966 \\
\hline $\operatorname{Pscd} 3$ & Pleckstrin homology, Sec7 and coiled-coil domains 3 & NM_053912.2 & 0.00242 & 48.629 \\
\hline Fgfl2 & Fibroblast growth factor 12 & NM_130814.1 & 0.00242 & 21.89 \\
\hline Kit & $\begin{array}{l}\text { v-Kit Hardy-Zuckerman } 4 \text { feline sarcoma viral oncogene } \\
\text { homolog }\end{array}$ & NM_022264.1 & 0.00242 & 32.155 \\
\hline \multicolumn{5}{|c|}{$\begin{array}{l}\text { Purine } \\
\text { metabolism }\end{array}$} \\
\hline Gmpr & Guanosine monophosphate reductase & NM_057188.1 & 0 & 63.736 \\
\hline Adcy8 & Adenylate cyclase 8 (brain) & NM_017142.1 & 0.00242 & 43.906 \\
\hline Adcy2 & Adenylate cyclase 2 (brain) & NM_031007.1 & 0 & 36.184 \\
\hline Adcy3 & Adenylate cyclase 3 & NM_130779.1 & 0.00242 & 25.177 \\
\hline$P d e 9 a$ & Phosphodiesterase 9A & NM_138543.1 & 0 & 360.045 \\
\hline \multicolumn{5}{|c|}{ Cell structure } \\
\hline Actnl & Actinin $\alpha 1$ & NM_031005.2 & 0.00242 & 30.32 \\
\hline Actn3 & Actinin $\alpha 3$ & NM_133424.1 & 0.00242 & 39.463 \\
\hline Cspg2 & Chondroitin sulphate proteoglycan 2 & XM_215451.4 & 0 & 360.045 \\
\hline Ptk2 & Protein tyrosine kinase 2 & NM_013081.1 & 0.00242 & 47.335 \\
\hline \multicolumn{5}{|c|}{ Transportation } \\
\hline Slc18a2 & Solute carrier family 18 (vesicular monoamine), member 2 & NM_013031.1 & 0.00242 & 29.17 \\
\hline Slc29a2 & Solute carrier family 29 (nucleoside transporters), member 2 & NM_031738.1 & 0.00242 & 31.183 \\
\hline Slc39a6 & Solute carrier family 39 (metal ion transporter), member 6 & NM_001024745.1 & 0.00121 & 111.965 \\
\hline Slc6a4 & $\begin{array}{l}\text { Solute carrier family } 6 \text { (neurotransmitter transporter, serotonin), } \\
\text { member } 4\end{array}$ & NM_013034.1 & 0.00242 & 50.804 \\
\hline Slc $9 a 5$ & Solute carrier family 9 (sodium/hydrogen exchanger), member 5 & NM_138858.1 & 0.00121 & 133.543 \\
\hline KIF4 & Kinesin family member 4 & XM_343797.3 & 0 & 360.045 \\
\hline \multicolumn{5}{|l|}{ Apoptosis } \\
\hline Anp32a & $\begin{array}{l}\text { Acidic (leucine-rich) nuclear phosphoprotein } 32 \text { family, } \\
\text { member A }\end{array}$ & NM_012903.1 & 0.00242 & 46.678 \\
\hline Orcl & Origin recognition complex, subunit 1 & NM_177931.2 & 0 & 360.045 \\
\hline Plag1 & Pleiomorphic adenoma gene 1 & NM_001008316.1 & 0 & 360.045 \\
\hline Mapk8 & Mitogen-activated protein kinase 8 & XM_341399.2 & 0.00242 & 28.211 \\
\hline$E 2 f 1$ & E2F transcription factor 1 & XM_230765.4 & 0.00121 & 130.672 \\
\hline Smo & Smoothened homolog (Drosophila) & NM_012807.1 & 0.00242 & 71.987 \\
\hline Ccnel & Cyclin E & XM_574426.1 & 0.00242 & 58.898 \\
\hline Actn3 & Actinin $\alpha 3$ & NM_133424.1 & 0.00242 & 39.463 \\
\hline $\operatorname{Inh} \alpha$ & Inhibin $\alpha$ & NM_012590.1 & 0.00242 & 55.708 \\
\hline Trim35 & Tripartite motif protein 35 & NM_001025142.1 & 0.00121 & 124.313 \\
\hline Phldal & Pleckstrin homology-like domain, family A, member 1 & NM_017180.1 & 0.00242 & 43.949 \\
\hline Sst & Somatostatin & NM_012659.1 & 0.00121 & 65.352 \\
\hline
\end{tabular}

a'Only genes with an explicit function are listed.

and centrioles. Kif2b, CLASP1 and SPAG5 (astrin) form a molecular switch in the centromere to regulate centromere and microtubule dynamics, promote the process of mitosis and maintain mitosis loyalty (21).

For the genes associated with transcription, high-mobility group AT-hook 1 (HMGA1) is a non-histone protein involved in numerous cellular processes, including the regulation of inducible gene transcription, integration of retroviruses into chromosomes and metastatic progression of cancer cells (22). The level of HMGA is high in embryonic tissues and insignificant in normal adult tissues. As early as ED 8.5, HMGA1 transcripts are highly detectable in all embryonic tissues. During mid-late gestation, its expression is gradually confined to specific body organs and tissues, including the central 
nervous system (primarily confined to the germinal layer), primordial liver, kidney and the retina. The high expression of the HMGAl gene is associated with cell proliferation and to establishing the correct identity of particular cells types (23). As a transcription regulatory factor, HMGA1 could stimulate the binding of nuclear factor- $\kappa \mathrm{B}$ to the interferon- $\beta$ promoter interacting with the p50 and p65 subunits. The HMGA1 proteins may be involved in organizing chromatin at the local level to provide the correct architecture for other transcription factors that are associated with playing a significant role in controlling growth.

The Foxm1 protein is a proliferation-specific member of the Fox family. Liver regeneration markedly induces Foxm1b expression at the G1/S transition and continues throughout hepatocyte proliferation.

Several signal transducers are also upregulated in rat embryonic liver. $P d e 9 a$, which maps to human chromosome 21q22.3 between TFF1 and D21S360, includes $>20$ splice variants, potentially changing the $\mathrm{N}$-terminal amino acid sequences of the encoded proteins. Pde9a is highly expressed in the brain, heart, placenta, adult and embryonic kidney, spleen, prostate and colon (24), has a high affinity for cyclic guanosine 3',5'-monophosphate (cGMP) and can catalyze the hydrolysis of cGMP to the corresponding nucleoside 5'-monophosphate.

In association with the cell structure, the protein Cspg2 belongs to the extracellular matrix chondroitin sulphate proteoglycan family. Cspg2 has four isoforms, labeled V0-V3. The isoforms V0 and V1 are highly expressed during embryonic development and their expression decreases following tissue maturation. Cspg2 is involved in extracellular matrix assembly and in controlling cell adhesion, proliferation, migration and apoptosis (25). In the mesenchymal cell condensation area, Cspg2 is highly expressed during the development of cartilage, heart, hair follicles and kidney. In vitro evidence shows that the Cspg 2 V0 and V1 isoforms are involved in precartilage mesenchymal condensation and subsequent chondrogenesis (26). The requirement of Cspg 2 in development is highlighted by the finding that a Cspg2 deficiency (in a transgenic mouse model) is embryonically lethal due to defects in cardiac formation, limb mesenchymal aggregation and chondrogenesis (27). However, the overexpression of Cspg2 in the embryonic liver has not been reported previously.

The group that consists of the genes associated with transportation includes a variety of genes involved in material transportation, such as solute carrier family 18, member 2 (Slc18a2), which transports vesicular monoamine; Slc29a2 (which transports nucleosides); Slc39a10, which transports zinc ions); Slc39a6, which transports metal ions; Slc6a4, which is a neurotransmitter transporter; Slc9a5, which is a $\mathrm{Na}^{+} / \mathrm{H}^{+}$ transporter protein; and KIF4, which transports cytoplasmic vesicles. The transport of these substances can regulate the activities of certain significant biological enzymes. Specific substances form cofactors and particular substances are involved in the signal transduction processes of growth factors or hormones.

Hepatogenesis requires the growth and proliferation of certain cells and the removal of others by apoptosis. However, only a limited number of genes associated with apoptosis were present on the chips in the present study. The pleomorphic adenoma gene 1 (Plagl) is a novel developmentally-regulated
$\mathrm{C}_{2} \mathrm{H}_{2}$ zinc finger gene. The deduced amino acid sequence of the Plag1 protein shows seven canonical $\mathrm{C}_{2} \mathrm{H}_{2}$ zinc finger domains and a serine-rich $\mathrm{C}$ terminus; the latter may have a regulatory function. According to Pendeville et al (28), the embryonic lung and liver, but not adult organs, primarily express Plag1. However, Plag1 has been found in a variety of tumors. The oncogenic activity of Plag1 results from its positive regulation of insulin growth factor 2 (IGF-II) expression. The peptide growth factor IGF-II plays an important role in embryonic development and carcinogenesis. The expression of IGF-II is primarily located in hepatocytes of the embryonic and neonatal liver (29). These findings indicate that the upregulation of Plag1 could reduce the apoptosis rate of cancer cells.

Orc1 is the largest subunit of the origin recognition complex (ORC), which plays a critical role during the initiation of DNA replication in eukaryotes. The expression level of the Orc1 subunit oscillates throughout the cell cycle (which defines an Orc1 cycle); it accumulates during the G1 phase and is degraded during the $\mathrm{S}$ phase. By controlling the progression of the $\mathrm{S}$ phase, Orc1 regulates the growth of animal cells. Orc1 is closely correlated with cell proliferation and the cell cycle and strictly controls the progress of the cell cycle. Saha et al (30) found that low Orc1 levels could rapidly induce caspase-3 activity, which subsequently induces p53-independent apoptosis. Orcl is expressed in the adult brain and muscle and in numerous embryonic tissues. However, no studies have been performed regarding its expression in the liver. The results of the present study show that Orcl may play a multifunctional role in the embryonic liver, and it possibly functions as a mediator of p53-independent apoptosis.

In conclusion, 11 upregulated genes were found in the ED 14 rat liver compared to the adult rat liver, which may provide novel insights into the molecular mechanisms that control hepatogenesis. These overexpressed genes are potential markers for identifying hepatic progenitor cells.

\section{Acknowledgements}

The present study was supported by the Shandong Province Natural Science Foundation (grant nos. ZR2010HM006, ZR2010HM065 and ZR2010HM087), the Shandong Province Higher Educational Science and Technology Program (grant nos. J07WE27 and J11LF14), the Shandong Province Medicine and Health Science Technology Program (grant no. 2013WS0279) and the Shandong Province Taishan Scholar Project.

\section{References}

1. Zaret KS: Hepatocyte differentiation: from the endoderm and beyond. Curr Opin Genet Dev 11: 568-574, 2001.

2. Houssaint E: Differentiation of the mouse hepatic primordium. I. An analysis of tissue interactions in hepatocyte differentiation. Cell Differ 9: 269-279, 1980.

3. Germain L, Blouin MJ and Marceau N: Biliary epithelial and hepatocytic cell lineage relationships in embryonic rat liver as determined by the differential expression of cytokeratins, alpha-fetoprotein, albumin, and cell surface-exposed components. Cancer Res 48: 4909-4918, 1988.

4. Petkov PM, Zavadil J, Goetz D, et al: Gene expression pattern in hepatic stem/progenitor cells during rat fetal development using complementary DNA microarrays. Hepatology 39: 617-627, 2004. 
5. Rossi JM, Dunn NR, Hogan BL and Zaret KS: Distinct mesodermal signals, including BMPs from the septum transversum mesenchyme, are required in combination for hepatogenesis from the endoderm. Genes Dev 15: 1998-2009, 2001.

6. Matsumoto K, Yoshitomi H, Rossant J and Zaret KS: Liver organogenesis promoted by endothelial cells prior to vascular function. Science 294: 559-563, 2001.

7. Jung J, Zheng M, Goldfarb $M$ and Zaret KS: Initiation of mammalian liver development from endoderm by fibroblast growth factors. Science 284: 1998-2003, 1999.

8. Sosa-Pineda B, Wigle JT and Oliver G: Hepatocyte migration during liver development requires Prox1. Nat Genet 25: 254-255, 2000.

9. Keng VW, Yagi H, Ikawa M, et al: Homeobox gene Hex is essential for onset of mouse embryonic liver development and differentiation of the monocyte lineage. Biochem Biophys Res Commun 276: 1155-1161, 2000.

10. Watt AJ, Garrison WD and Duncan SA: HNF4: a central regulator of hepatocyte differentiation and function. Hepatology 37 : 1249-1253, 2003.

11. Zhao R, Watt AJ, Li J, Luebke-Wheeler J, Morrisey EE and Duncan SA: GATA6 is essential for embryonic development of the liver but dispensable for early heart formation. Mol Cell Biol 25: 2622-2631, 2005

12. Uppalapati D, Ohta N, Zhang Y, et al: Identification and characterization of unique tumoricidal genes in rat umbilical cord matrix stem cells. Mol Pharm 8: 1549-1558, 2011.

13. Chen YF, Pottala JV, Weltman NY, Ge X, Savinova OV and Gerdes AM: Regulation of gene expression with thyroid hormone in rats with myocardial infarction. PLoS One 7: e40161, 2012.

14. Dabeva MD, Petkov PM, Sandhu J, Oren R, Laconi E, Hurston E and Shafritz DA: Proliferation and differentiation of fetal liver epithelial progenitor cells after transplantation into adult rat liver. Am J Pathol 156: 2017-2031, 2000.

15. Jiao X, Sherman BT, Huang da W, Stephens R, Baseler MW, Lane HC and Lempicki RA: DAVID-WS: a stateful web service to facilitate gene/protein list analysis. Bioinformatics 28 1805-1806, 2012.

16. Hosack DA, Dennis G Jr, Sherman BT, Lane HC and Lempicki RA: Identifying biological themes within lists of genes with EASE. Genome Biol 4: R70, 2003.

17. Nishimura M and Naito S: Tissue-specific mRNA expression profiles of human carbohydrate sulfotransferase and tyrosylprotein sulfotransferase. Biol Pharm Bull 30: 821-825, 2007.

18. Pancholi V: Multifunctional alpha-enolase: its role in diseases. Cell Mol Life Sci 58: 902-920, 2001.
19. D'Angiolella V, Donato V, Vijayakumar S, et al: SCF(Cyclin F) controls centrosome homeostasis and mitotic fidelity through CP110 degradation. Nature 466: 138-142, 2010.

20. Du J, Jablonski S, Yen TJ and Hannon GJ: Astrin regulates Aurora-A localization. Biochem Biophys Res Commun 370 213-219, 2008

21. Mack GJ and Compton DA: Analysis of mitotic microtubule-associated proteins using mass spectrometry identifies astrin, a spindle-associated protein. Proc Natl Acad Sci USA 98: 14434-14439, 2001.

22. Ben-Porath I, Thomson MW, Carey VJ, Ge R, Bell GW, Regev A and Weinberg RA: An embryonic stem cell-like gene expression signature in poorly differentiated aggressive human tumors. Nat Genet 40: 499-507, 2008.

23. Chiappetta G, Avantaggiato V, Visconti R, et al: High level expression of the HMGI (Y) gene during embryonic development. Oncogene 13: 2439-2446, 1996.

24. Rentero $\mathrm{C}$, Monfort $\mathrm{A}$ and Puigdomènech $\mathrm{P}$ : Identification and distribution of different mRNA variants produced by differential splicing in the human phosphodiesterase 9A gene. Biochem Biophys Res Commun 301: 686-692, 2003.

25. Theocharis AD: Versican in health and disease. Connect Tissue Res 49: 230-234, 2008.

26. Kamiya N, Watanabe H, Habuchi H, Takagi H, Shinomura T, Shimizu K and Kimata K: Versican/PG-M regulates chondrogenesis as an extracellular matrix molecule crucial for mesenchymal condensation. J Biol Chem 281: 2390-2400, 2006.

27. Williams DR Jr, Presar AR, Richmond AT, Mjaatvedt $\mathrm{CH}$, Hoffman S and Capehart AA: Limb chondrogenesis is compromised in the versican deficient hdf mouse. Biochem Biophys Res Commun 334: 960-966, 2005.

28. Pendeville H, Peers B, Kas K and Voz ML: Cloning and embryonic expression of zebrafish PLAG genes. Gene Expr Patterns 6: 267-276, 2006

29. Voz ML, Agten NS, Van de Ven WJ and Kas K: PLAG1, the main translocation target in pleomorphic adenoma of the salivary glands, is a positive regulator of IGF-II. Cancer Res 60: 106-113, 2000 .

30. Saha T, Ghosh S, Vassilev A and DePamphilis ML: Ubiquitylation, phosphorylation and Orc2 modulate the subcellular location of Orc1 and prevent it from inducing apoptosis. J Cell Sci 119: 1371-1382, 2006. 